\title{
An Unusual Finding of Buschke-Löwenstein Tumor Associated with 6, 39 and 53 HPV Genotypes in a Young Immunocompetent Female
}

\author{
Peronace $C^{1^{*}}$, Galati $L^{1}$, Barreca GS ${ }^{1}$, Lamberti AG ${ }^{1}$, Curcio $B^{2}$, Morelli $M^{2}$, Conforti $F^{3}$, Matera $\mathbf{G}^{1}$, Liberto MC ${ }^{1}$, Zullo $F^{2}$ and Focà $A^{1}$ \\ ${ }^{1}$ Department of Health Sciences, Institute of Microbiology, School of Medicine, University "Magna Graecia”, Catanzaro, Viale Europa, Catanzaro, Italy \\ ${ }^{2}$ Department of Experimental and Clinical Medicine, Unit of Obstetrics and Gynecology, University "Magna Graecia”, Catanzaro , Viale Europa, Catanzaro, Italy \\ 3Departmentof Health Sciences, University "Magna Graecia”, Viale Europa, Catanzaro, Italy
}

"Corresponding author: Peronace C, Department of Health Sciences, Institute of Microbiology, School of Medicine, University "Magna Graecia”, Catanzaro, Viale Europa, Catanzaro, Italy, Tel: +39-0961-3697743 Fax: +39-0961-3697760; E-mail: cinziaperonace@hotmail.it

Received date: October 17, 2016; Accepted date: November 03, 2016, Published date: November 20, 2016

Copyright: (C) 2016 Peronace C, et al. This is an open-access article distributed under the terms of the Creative Commons Attribution License, which permits unrestricted use, distribution, and reproduction in any medium, provided the original author and source are credited.

\begin{abstract}
Background: Buschke-Löwenstein tumor (BLT) or giant condyloma acuminatum (GCA), is a rare disease caused by Human Papilloma virus (HPV) types 6 and 11, more common in immunocompromised males. We describe a rare case of BLT associated with mixed low and high-risk HPV type's infection in a very young immunocompetent female.

Methods: On August 2012, an 18-year old female was admitted to the Unit of Obstetrics and Gynecology, with a 3-4 month history of small excrescences in the perineal/vulvar area which had rapidly increased in size. A diagnosis of BLT was suspected; an incisional biopsy was carried out. On the cervical and vulvar cytobrush samples, Polymerase Chain Reaction (PCR) of specific sequences of the HPV genome L1 region was performed. Surgical treatment was carried out by cold knife excision.
\end{abstract}

Results: Clinically, the tumor presented as exophytic white lesion, characterized by an irregular surface with acuminate excrescenses. Microscopic images are consistent with diagnosis of Buschke-Lowenstein tumor. Performed PCR revealed the presence of HPV types 6, 39 and 53. After surgery the patient showed no signs of recurrence during the 4 years of follow-up.

Conclusions: This report a rare case of BLT in a young immunocompetent female, associated with multiple HPV type infection. The studied case suggests that pathologies with a BLT-like clinical and histological presentation and mixed HPV genotypes can be found in young immunocompetent patient.

Keywords: Buschke-Löwenstein tumor; Multiple human papillomavirus infection; Immunocompetent patient

List of abbreviations Buschke-Löwenstein Tumor (BLT); Giant Condyloma Acuminatum (GCA); Human Papilloma Virus (HPV); Polymerase Chain Reaction (PCR); Squamous Cell Carcinoma (SCC); Linear Probe Assay (LiPA).

\section{Introduction}

The Buschke-Löwenstein tumor (BLT) or giant condyloma acuminatum (GCA) is a very rare, sexually transmitted disorder frequently located in the anogenital region, first described in 1925 [1], comprising no more than $0.1 \%$ of anogenital warts [2]. Usually, BLT is characterized by a slowly growing cauliflower-like lesion, triggered by Human Papilloma virus (HPV) infection, most commonly low-risk HPV genotypes 6 and 11 [3] and occasionally types 16 and 18 [4]. Buschke-Löwenstein tumor mostly affects middle-aged man (reportedly 43 years), with a 2.7:1 male-to-female ratio; although it shows a benign behavior, a tendency to infiltrate the adjacent tissues and to transform into squamous cell carcinoma (SCC) is still possible. Due to a relevant recurrence rate (66\%), a regular follow-up is necessary [5,6]. A severely compromised immune system represents the most important risk factor for development of genital lesions, potentially favoring the oncogenetic mechanisms triggered by HPV infection. Case presentations of BLT were frequently reported in immunocompromised patients [7-9]. On the contrary, we present a case of Buschke-Löwenstein tumor bearing its uniqueness due to: i) the young age of the patient, ii) the female sex, iii) her immunocompetent condition and, iv) the multiple low and high risk HPV types infection.

\section{Materials and Methods}

On August 2012, an 18-year old Italian female was admitted to the Unit of Obstetrics and Gynecology of the University Hospital of Catanzaro (Italy), with a 3-4 months history of small excrescences in the perineal/vulvar area, which had rapidly increased in size. The patient did not have a significant past medical history and did not refer previous relevant interventions. Physical examination included colposcopy and vulvoscopy. Vulvoscopic evaluation highlighted an exophytic white lesion, characterized by an irregular surface with acuminate excrescenses. The lesion was localized on vulvar fourquette, extending laterally to the labia majora for about $4-5 \mathrm{~cm}$, in the cranial direction and caudally, along the midline, until near the anus (Figure 1). Colposcopic evaluation showed no evidences of cervical disease. A diagnosis of BLT was suspected. Differential diagnosis included condyloma acuminatum. 
Citation: Peronace C, Galati L, Barreca GS, Lamberti AG, Curcio B, et al. (2016) An Unusual Finding of Buschke-Löwenstein Tumor Associated with 6, 39 and 53 HPV Genotypes in a Young Immunocompetent Female. Clin Microbiol 5: 265. doi:10.4172/2327-5073.1000265

Page 2 of 4

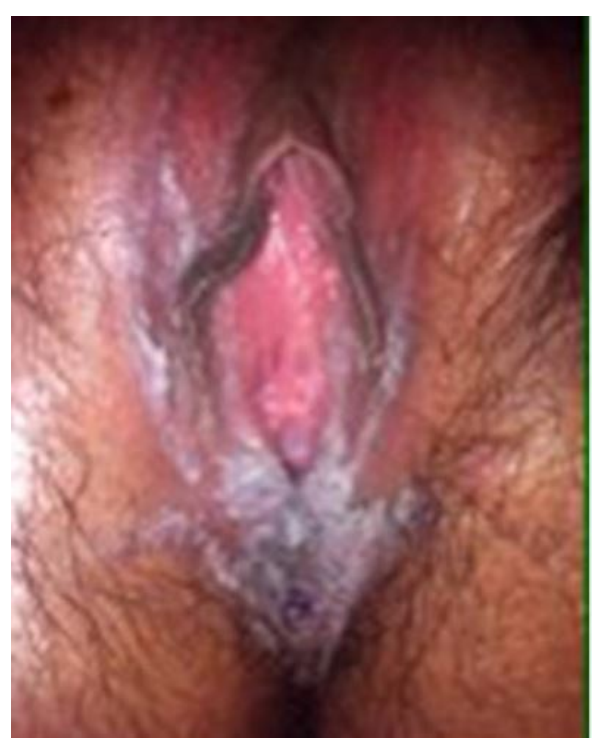

Figure 1: Clinical presentation of the studied case of BuschkeLöwenstein disease. The lesion was localized on vulvar fourquette, extending laterally to the labia majora for about $4-5 \mathrm{~cm}$, in the cranial direction and caudally, along the midline, until near the anus.

Cervical and vulvar brushing specimens were separately carried out for PCR and HPV genotyping. Vulvar brushing specimens were carried out by thin cytobrush to ensure that the sampling was from our lesion, without any contamination from the surrounding areas. Cervical brushing was carried out in standard way. DNA extraction was performed by automated method using EasyMag (bioMérieux, Italy). Eluted DNA $(10 \mu \mathrm{l})$ was used for the PCR amplification of HPV sequences from the L1 region using an amplification kit (INNO-LiPA HPV Genotyping Extra Amp, Innogenetics, Belgium). Particularly the test uses 5'-biotynilated SPF10 primers to amplify a 65 bp fragment within the L1 open reading frame in HPV genome. An additional primer pair for the amplification of the human $H L A-D P B 1$ gene is added to monitor sample quality, extraction and amplification. The resulting biotinylated amplicons are then denatured and hybridized with specific oligonucleotide probes using a single typing strip on which 28 sequence-specific DNA probes and 4 control lines are fixed. After drying the strips, the resulting purple precipitates were visually interpreted according to manufacturer's protocol by using an interpretation grid supplied with the kit (INNO-LiPA HPV Genotyping Extra, Innogenetics, Belgium) [10]. PCR and genotyping methods revealed the presence of HPV types 6, 39 and 53, in both cervical and vulvar brushing specimens. Moreover, an incisional biopsy, ( $5 \mathrm{~mm}$ large and $2 \mathrm{~mm}$ deep) with a share of stromal tissue was performed for histological examination. Microscopic evaluation showed exophytic component with hypercheratosis and parakeratosis, endophytic component and squamous cells with increased mitotic activity. Biopsy specimens embedded in paraffin for histological diagnosis, were also utilized for detection of HPV genotypes; briefly, each microscopic slide was deparaffinized with a $1 \mathrm{ml}$ of xylene and incubated at $50^{\circ} \mathrm{C}$ for $5 \mathrm{~min}$. The tissue was pelleted at $14,000 \mathrm{x} \mathrm{g}$ for 2 min and the resultant pellet washed twice with $1 \mathrm{ml}$ of $95 \%-100 \%$ ethanol and was air dried for $10 \mathrm{~min}$ to ensure that no residual ethanol was present. DNA extraction was performed according to the manufacturer's instruction for a QIAamp DNA Mini Kit (QIAGEN, Germany) and the final eluted DNA was utilized for PCR and HPV genotyping using LiPA method, as previously described. PCR and genotyping methods revealed the presence of HPV types 6, 39. Routine laboratory tests showed no abnormalities. Antibodies against HIV, $\mathrm{HCV}$ and HBV were negative. In view of the extension of the lesion near to anus, the patient underwent to preliminary rectoscopy, which excluded the involvement of rectal mucosa. Before surgery, a broad spectrum antibiotic was administered (ceftriaxone). Complete surgical removal was carried out by cold knife excision, in multiple incisions in order to preserve more healthy tissue as possible, with wide margins of resection and reconstruction of the vulva with interrupted sutures by Vycril4-0 (Figures 1 and 2).

Histopathological examination of the resected tissue, revealed massive epidermal hyperplasia, hyperkeratosis and parakeratosis (Figure 3a). Some keratinocytes had nucleus with prominent nucleoli and large cytoplasm. Keratinocytes with granular vacuolization exhibited large cytoplasm and nucleus with large prominent and visible nucleoli. Tumor cells have discrete cytoplasmic nuclear atypia (Figure $3 b)$, but they were not detected in blood or lymphatic vessels. Rounded masses are also observed that invade the dermis and contiguous structures (Figure 3c). Condyloma acuminatum was excluded because histopathological images thickening of the stratum corneum, endophytic increases and deeper invasion argues for giant condyloma. Final histopathology findings were consistent with the diagnosis of Buschke-Löwenstein tumor. Adverse events and infective complications were not observed. It was performed a dressing after 10 days of surgery; the first follow-up programmed to one month showed the restitutio and integrum. After radical surgical excision, the patient showed no local recurrence signs during four years of follow-up. This study was approved by the Catanzaro University Hospital Ethical Committee in compliance with the Declaration of Helsinki. Informed written consent was obtained by the patient for publication of this case report and any accompanying images.

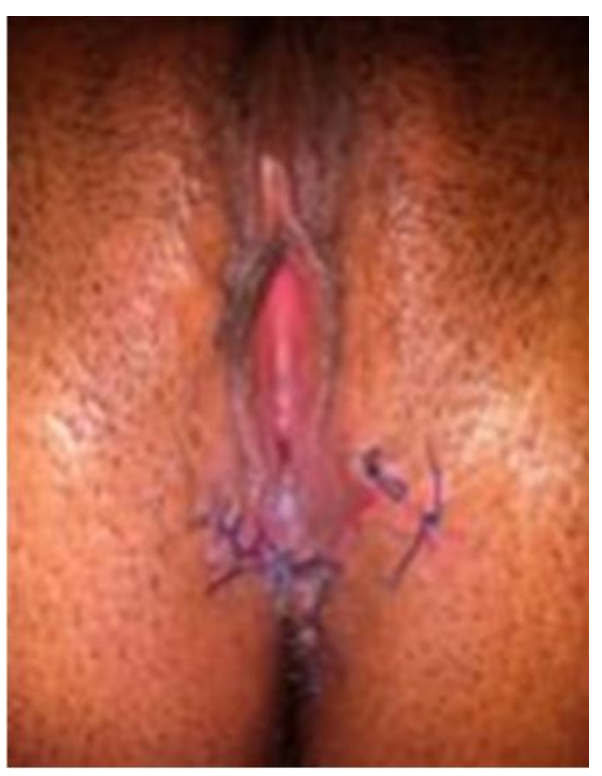

Figure 2: Genital appearance after treatment. Reconstruction of the vulva after cold knife surgical excision. 


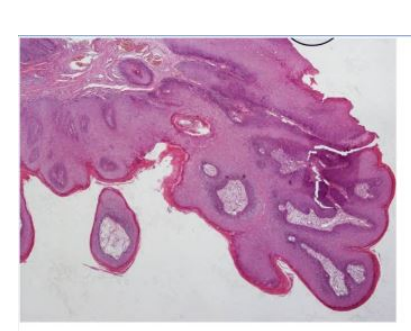

a

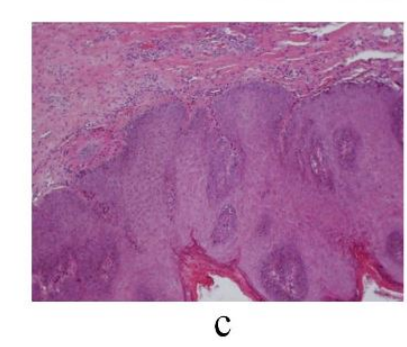

Figure 3: Photomicrographs of the excised tissue. (a) Hyperkeratosis, and parakeratosis (HE staining, X4); (b) The arrows indicate squamous cells with increased mitotic activity (HE staining, X40). (c) Finger-like processes with absence of basement membrane involvement (HE staining, X10)

\section{Conclusions}

Buschke-Löwenstein tumor (BLT) or giant condyloma acuminate (GCA), reported as a rare sexually transmitted disease, and is a large, exophytic lesion, with a cauliflower-like morphology, frequently located in the anogenital region. In our case, the lesion did not show the characteristics proliferation frequently described in the BLT disease, due to the early diagnosis of our BLT case. Therefore, evaluation of the internal anal canal should be done before starting the treatment. In this case, the preoperative rectoscopy revealed no relevant findings. In investigated patient no evidence of malignancy appeared and there is no evidence of recurrence in the three postoperative years. The role of human papilloma virus infection in the etiopathogenesis of BLT has been widely established. Indeed low-risk HPV types 6 and 11 were commonly associated to BLT disease.

On the contrary, we found a multiple infection due to HPV types 6, 39 and 53. Despite the outcome of PCR amplification from paraffinembedded specimens on microscopic slide can be hindered, we still detected genotypes 6 and 39, as result of multiple infections. Immunosuppression is one of the risk factors for BLT development, nevertheless our patient was immunocompetent, but a rapid growth of the lesion had been observed. The strengths of this approach to this case, include the timely diagnosis and surgical excision, as a well as the virological assessment of the HPV etiology. The availability of vaccinations against HPV infection is contributing to the decreasing incidence of this infection. The purpose of this case suggests that pathologies with a BLT-like clinical and histological presentation and multiple HPV types, though in young immunocompetent patient, must be timely managed and carefully followed as in older/ immunosuppressed patients.

In conclusion, this reported is a rare case of BLT in a young immunocompetent female, associated with multiple HPV type infection. Further studies are needed to define this disease and address its pathogenesis.

\section{Authors' Contributions}

CP: conceived the study, drafted the manuscript and participated in its design. LG contributed in the draft the manuscript and carried out molecular assay. GSB carried out and supervised molecular assay. AGL contributed to the molecular biology test and discussed corresponding data. BC contributed to patient assessment and collected clinical data. MM took care the patient during hospitalization and contributed to the draft of the manuscript. FC carried out the pathology procedures and contributed to the manuscript revision. GM contributed to the draft and editing of the manuscript. MCL coordinated virological assessments and contributed in the draft and editing of the manuscript. FZ contributed to diagnosis and performed surgical intervention. AF conceived the study and participated in its design and coordination. All authors read and approved the final manuscript.

\section{Ethics Approval and Consent to Participate}

This study was approved by the Catanzaro University Hospital Ethical Committee in compliance with the Declaration of Helsinki. Informed written consent was obtained by the patient for publication of this case report and any accompanying images.

\section{Competing Interests}

We declare that we have no conflict of interest

\section{Acknowledgement}

Financial support for this case report was entirely provided by the University of Catanzaro (Grant: RICEFOC-01; Funder: University of Catanzaro; Recipient: Prof. Alfredo Focà).

\section{References}

1. Buschke A, Löwenstein L (1925) Überkarzinom- ähnliche Condylomata acuminata des Penis Klin Wochenschr. 4: 1726:1728.

2. Akhavizadegan H (2015) Electrocautery Resection, Shaving with a Scalpel, and Podophyllin: a Combination Therapy for Giant Condyloma Acuminatum. World J Mens Health 33: 39:41.

3. Ahsaini M, Tahiri Y, Tazi MF, Elammari J, Mellas S, et al. (2013) Verrucous carcinoma arising in an extended giant condyloma acuminatum (Buschke-Löwenstein tumor): a case report and review of the literature. J Med Case Rep 7: 273.

4. Ciobanu AM, Popa C, Marcu M, Ciobanu CF (2014) Psychotic depression due to giant condyloma Buschke-Löwenstein tumors. Rom J Morphol Embryol 55: 189-195.

5. Tas S, Arik MK, Ozkul F, Cikman O, and Akgun Y (2012) Perianal giant condyloma acuminatum-Buschke- Löwenstein tumor: A case report. Case Reports in Surgery Volume 2012.

6. Mestrovic T, Cavcic J, Martinac P, Turcic J, Zupancic B, et al. (2003) Reconstruction of skin defects after radical excision of anorectal giant condyloma acuminatum: 6 cases. J Eur Acad Dermatol Venereol 17:541-545.

7. Wester NE, Hutten EM, Krikke C, Pol RA (2013) Intra-abdominal localisation of a Buschke-Löwenstein tumour: case presentation and review of the literature. Case Rep Transplant Volume 2013.

8. Chu QD, Vezeridis MP, Libbey NP, Wanebo HJ, et al. (1994) Giant condyloma acuminatum (BuschkeLowenstein tumor) of the anorectal and perianal regions: analysis of 42 cases. Diseases of the Colon and Rectum 37:950-57.

9. Trottier H, Burchell AN (2009) Epidemiology of mucosal human papilloma virus infection and associated diseases. Public Health Genomic 12: 291-07. 
Citation: Peronace C, Galati L, Barreca GS, Lamberti AG, Curcio B, et al. (2016) An Unusual Finding of Buschke-Löwenstein Tumor Associated with 6, 39 and 53 HPV Genotypes in a Young Immunocompetent Female. Clin Microbiol 5: 265. doi:10.4172/2327-5073.1000265

Page 4 of 4

10. Galan-Sanchez F, Hernández-Menendez M, De Los Rios Hernandez MA, Rodriguez-Iglesias M (2011) Performance of the New INNO-LiPA HPV extra to genotype human papillomavirus in cervical cell specimens. Acta Cytol 55: 341-343. 